\title{
Evaluation of New Ceramics as Column Packing Material for High Performance Liquid Chromatography
}

\author{
Masahiro Kawahara, Hiroshi Nakamura and Terumi Nakajima \\ Faculty of Pharmaceutical Sciences, University of Tokyo, Hongo, Tokyo 113
}

\begin{abstract}
Keywords Silica gel, zirconia, new ceramics, column packing, high performance liquid chromatography, Freundrich equation
\end{abstract}

Silica gel and its derivatives have been predominant column packing materials for modern liquid chromatography owing to their high resolution. ${ }^{1}$ However, their use is limited to the $\mathrm{pH}$ range 2.5 to 7.5 due to the chemical instability of their silica gel matrixes. ${ }^{2}$ Nowadays, various type of new materials, i.e., new ceramics, are easily available and their beneficial characteristics are widely known. ${ }^{3}$ On account of their ability to resist chemicals, heat and physical shocks, these new ceramics have been used as key materials for various devices in industries. On the other hand, the characteristics of these new materials indicate they should be usable as promising packing materials for high performance liquid chromatography (HPLC) as well. Therefore, we evaluated the adsorptive properties of some new ceramics, as a column packing material for HPLC, by using a newly developed method.

\section{Experimental}

\section{Materials}

Reagents and solvents were of guaranteed grade. Silica gel 60 for column chromatography (particle size, $0.063-0.200 \mathrm{~mm})$, LiChrosorb Si-60 $(5 \mu \mathrm{m})$, and aluminum oxide 90 active neutral for column chromatography $(0.063-0.200 \mathrm{~mm})$ were purchased from $\mathrm{E}$. Merck (Darmstadt, West Germany). Wakogel C-200 $(0.074-0.149 \mathrm{~mm})$ and zirconium silicate were purchased from Wako Pure Chemical Industries Ltd. (Osaka, Japan). Zirconium nitride $(1.35 \mu \mathrm{m})$ and zirconium carbide $(0.97 \mu \mathrm{m})$ were kindly donated from Japan New Metals Co. Ltd. (Osaka, Japan); titanium oxide (1 $2 \mu \mathrm{m})$, zirconium oxide $(3 \mu \mathrm{m})$ and zirconium oxyphosphate were purchased from Soekawa Chemical Co., Ltd. (Tokyo, Japan).

\section{Measurement of adsorption isotherms}

The determination of the adsorption isotherms was carried out as follows. Twenty mg of the adsorbent was placed in individual test tubes and dried at $105^{\circ} \mathrm{C}$ for
$2 \mathrm{~h}$. A series of $p$-hydroxybenzoic acid ( $p$-HBA) solutions of various concentrations were prepared with hexane-2-propanol $(9: 1)$. To each test tube containing the adsorbent, $500 \mu \mathrm{l}$ of each solution of $p$-HBA was added. After ultrasonication, the tubes were shaken rapidly at $40^{\circ} \mathrm{C}$ for $1 \mathrm{~h}$. Thereafter the slurry was centrifuged, and the concentration of $p$-HBA in the supernatant $\left(C_{\mathrm{m}} ; \mu \mathrm{M}\right)$ was measured by the following reversed-phase HPLC.

\section{HPLC}

A TRIROTAR-VI (JASCO, Tokyo, Japan) HPLC system was used. $p$-HBA was separated on a TSK gel ODS-80 $\mathrm{T}_{\mathrm{M}}$ column $(4.6 \mathrm{~mm}$ i.d. $\times 150 \mathrm{~mm}, 5 \mu \mathrm{m}$, Tosoh, Tokyo, Japan) with isocratic elution using $0.02 \% \mathrm{HCl}-$ acetonitrile (1:4) and detected at $254 \mathrm{~nm}$.

\section{Results and Discussion}

There are several methods for testing performance of adsorbents; the shake-flask method ${ }^{4}$ is rapid and easy to control conditions, while it requires no special apparatus. Therefore, the adsorption isotherms were determined for $p$-HBA in hexane-2-propanol $(9: 1)$ on various new ceramics using the shake-flask method.

The amount of $p$-HBA adsorbed per weight of the adsorbent $\left(C_{\mathrm{s}} ; \mathrm{nmol} / \mathrm{mg}\right)$ was calculated from the following relationship

$$
C_{\mathrm{s}}=\left(C_{0}-C_{\mathrm{m}}\right) \times V / W
$$

where $C_{0}(\mu \mathrm{M})$ is the initial concentration of $p$-HBA, $C_{\mathrm{m}}(\mu \mathrm{M})$ is the concentration of $p$-HBA in the supernatant, $V(\mathrm{ml})$ is the volume of the solution, and $W$ (mg) is the weight of the adsorbent. Figure 1 shows typical adsorption isotherms on silica gel (LiChrosorb $\mathrm{Si}-60$ ), aluminum oxide, zirconium oxide and titanium oxide.

To represent the adsorption isotherms in liquid phase either Henry equation, Langmuir equation or Freundrich 
equation is commonly used. 5,6 The Henry equation (Eq. (2)) fits best for dilute solutions:

$$
C_{\mathrm{s}}=K_{\mathrm{d}} \times \mathrm{C}_{\mathrm{m}}
$$

The parameter $K_{d}(1 / g)$ is a distribution constant. The Langmuir equation (Eq. (3)) incorporates the assumptions of monolayer adsorption and uniformity of adsorption sites:

$$
C_{\mathrm{s}}=A \times Q_{\mathrm{m}} \times C_{\mathrm{m}} /\left(1+A \times C_{\mathrm{m}}\right)
$$

$A$ and $Q_{\mathrm{m}}$ (nmol/mg) represent the affinity and a maximum capacity of the adsorbent. To determine the

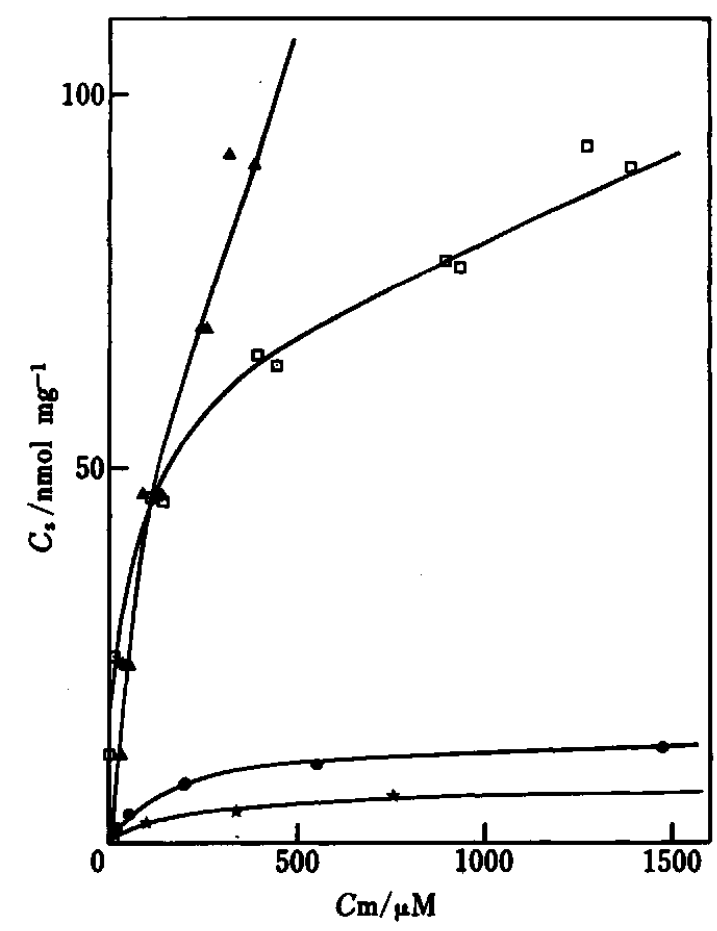

Fig. 1 Adsorption isotherms on inorganic oxides: ( $\Delta$ ) aluminum oxide; (ㅁ) zirconium oxide; $(\star)$ titanium oxide; (Ө) silica gel (LiChrosorb Si-60, $5 \mu \mathrm{m}$ ). adsorption parameters, two linearized forms (Eq. (3a), (3b)) of the Langmuir equation are employed:

$$
\begin{aligned}
& C_{\mathrm{m}} / C_{\mathrm{s}}=\left(1 / Q_{\mathrm{m}}\right) \times C_{\mathrm{m}}+1 /\left(A \times Q_{\mathrm{m}}\right) . \\
& 1 / C_{\mathrm{s}}=(1 / A) \times\left(1 / Q_{\mathrm{m}}\right) \times\left(1 / C_{\mathrm{m}}\right)+1 / Q_{\mathrm{m}} .
\end{aligned}
$$

The Freundrich equation (Eq. (4)) is an empirical equation:

$$
C_{\mathrm{s}}=K \times C_{\mathrm{m}}^{1 / n}
$$

The parameter $K$ is an adsorption capacity when $C_{\mathrm{m}}=1$. The parameter $n$ is related to the adsorption activity. The adsorption becomes firmer as $n$ increases. Generally the adsorbents with $n$ values of $2-5$ are used.

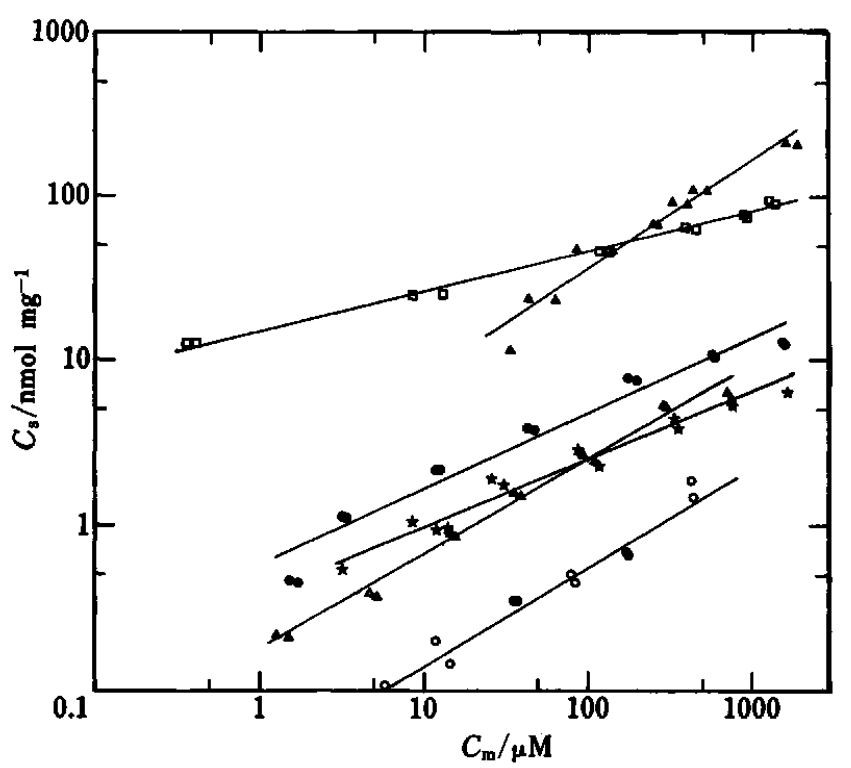

Fig. 2 Adsorption isotherms plotted on $\log -\log$ graph: $(\Delta)$ aluminum oxide; (ㅁ) zirconium oxide; (๑) LiChrosorb

\begin{tabular}{|c|c|c|c|c|c|c|c|c|c|c|c|}
\hline \multirow[b]{2}{*}{ Adsorbent } & \multicolumn{2}{|c|}{ Henry (Eq. (2)) } & \multicolumn{3}{|c|}{ Langmuir (Eq. (3a)) } & \multicolumn{3}{|c|}{ Langmuir (Eq. (3b)) } & \multicolumn{3}{|c|}{ Freundrich (Eq. (4)) } \\
\hline & $K_{\mathrm{d}} / 1 \mathrm{~g}^{-1}$ & $r$ & $\boldsymbol{A}$ & $\begin{array}{c}Q_{\mathrm{m}} / \\
\mathrm{nmol} \mathrm{mg}^{-1}\end{array}$ & $r$ & $\boldsymbol{A}$ & $\begin{array}{c}Q_{\mathrm{m}} / \\
\mathrm{nmol} \mathrm{\textrm { } \mathrm { g } ^ { - 1 }}\end{array}$ & $r$ & $\boldsymbol{K}$ & $n$ & $r$ \\
\hline Wakogel C-200 & $3.50 \times 10^{-2}$ & 0.980 & $2.11 \times 10^{-2}$ & $8.45 \times 10^{-1}$ & 0.974 & $4.64 \times 10^{-3}$ & 2.17 & 0.830 & $3.29 \times 10^{-2}$ & 1.61 & 0.985 \\
\hline Silica gel 60 & $9.43 \times 10^{-3}$ & 0.950 & $8.48 \times 10^{-2}$ & 1.96 & 0.957 & $7.66 \times 10^{-3}$ & 8.04 & 0.979 & $1.73 \times 10^{-1}$ & 1.72 & 0.968 \\
\hline LiChrosorb Si-60 & $7.77 \times 10^{-3}$ & 0.874 & $3.62 \times 10^{-2}$ & 8.89 & 0.986 & $1.18 \times 10^{-2}$ & 8.04 & 0.996 & $5.67 \times 10^{-1}$ & 2.16 & 0.980 \\
\hline $\mathrm{TiO}_{2}$ & $3.45 \times 10^{-3}$ & 0.892 & $2.40 \times 10^{-2}$ & 4.83 & 0.987 & $7.09 \times 10^{-3}$ & 7.40 & 0.991 & $3.56 \times 10^{-1}$ & 2.38 & 0.976 \\
\hline $\mathrm{Al}_{2} \mathrm{O}_{3}$ Active 90 & $1.04 \times 10^{-1}$ & 0.953 & $1.48 \times 10^{-3}$ & 295 & 0.956 & $1.47 \times 10^{-3}$ & 284 & 0.975 & 1.78 & 1.52 & 0.973 \\
\hline $\mathrm{ZrO}_{2}$ & $5.17 \times 10^{-2}$ & 0.926 & $8.57 \times 10^{-1}$ & 50.8 & 0.856 & $1.26 \times 10^{-2}$ & 90.6 & 0.989 & 15.0 & 4.14 & 0.997 \\
\hline $\mathrm{ZrSiO}_{4}$ & $3.51 \times 10^{-2}$ & 0.936 & $9.21 \times 10^{-1}$ & 4.43 & 0.948 & $6.69 \times 10^{-3}$ & 8.32 & 0.962 & 1.66 & 3.48 & 0.974 \\
\hline $\mathrm{ZrO} \cdot\left(\mathrm{H}_{2} \mathrm{PO}_{4}\right)$ & $6.29 \times 10^{-3}$ & 0.959 & $1.89 \times 10^{-3}$ & 7.23 & 0.989 & $3.65 \times 10^{-3}$ & 4.46 & 0.916 & $2.52 \times 10^{-2}$ & 1.23 & 0.974 \\
\hline $\mathrm{ZrN}$ & $9.52 \times 10^{-3}$ & 0.959 & $3.10 \times 10^{-2}$ & 4.72 & 0.919 & $1.03 \times 10^{-2}$ & 8.18 & 0.977 & $3.13 \times 10^{-1}$ & 2.00 & 0.971 \\
\hline $\mathrm{ZrC}$ & $1.65 \times 10^{-2}$ & 0.951 & $8.30 \times 10^{-2}$ & 1.99 & 0.864 & $5.43 \times 10^{-3}$ & 10.6 & 0.743 & $1.71 \times 10^{-1}$ & 1.61 & 0.964 \\
\hline
\end{tabular}
Si-60; $(\star)$ titanium oxide; $(\Delta)$ Silica gel Si-60; $(O)$ Wakogel $\mathrm{C}-200$. Solid lines represent those by the Freundrich equation for each adsorbent.

Table 1 Summary of isotherm equation parameters 
Equation (5) is derived from the logarithmic transformation of Eq. (4) and predicts logarithmic proportionality between $C_{\mathrm{s}}$ and $C_{\mathrm{m}}$ :

$$
\log C_{\mathrm{s}}=(1 / n) \log C_{\mathrm{m}}+\log K .
$$

The best-fit estimates of parameters and correlation coefficients of determination $(r)$ were calculated and compared with each adsorbent (Table 1). Although the two Langmuir equations gave better $r$ values than those obtained by the Henry equation, they showed rather poor representations over a wide range of solvent concentration. Equation (3a) fitted when $C_{\mathrm{m}}$ was high, while Eq. (3b) fitted when $C_{\mathrm{m}}$ was low. The Freundrich equation consistently gave good $r$ values close to unity. The typical $\log$ - $\log$ plots between $C_{\mathrm{s}}$ and $C_{\mathrm{m}}$ obtained with some adsorbents are shown in Fig. 2.

Thus the adsorption isotherms on various adsorbents were found to be well represented by the Freundrich equation. These adsorption capacities were usable for the evaluation of the new ceramics as a column packing material for HPLC. These findings clearly show that the method presented here will be useful in choosing a column packing material for use in HPLC. Detailed investigations will be reported elsewhere in the near future.

We thank Japan New Metals Co. Ltd. for giving us some new ceramics.

\section{References}

1. W. S. Hancock and J. T. Sparrow, "HPLC Analysis of Biological Compounds", Marcel Dekker, Inc., New York (1984).

2. J. L. Glajch, J. J. Kirkland and J. Kohler, J. Chromatogr., 384, 81 (1987).

3. G. Adachi, K. Sibayama and T. Minami, ed., "Sentanbronya ni okeru Zairyou Gijyutu", Kagaku Doujin, Kyoto (1984).

4. J.-X. Huang and C. Horváth, J. Chromatogr., 406, 285 (1987).

5. P. K. Gessner and M. M. Hasan, J. Pharm. Sci., 76, 319 (1987).

6. G. K. C. Low and G. E. Batley, J. Chromatogr., 355, 177 (1986).

(Received October 11, 1988)

(Accepted October 20, 1988) 